\title{
Magnetic giant magnetoresistance commercial off the shelf for space applications
}

\author{
Michelena, M.D.; Oelschlägel, Wulf; Arruego, I.; del Real, R.P; Mateos, J.A.; Merayo, José M.G.
}

Published in:

Journal of Applied Physics

Link to article, DOI:

$10.1063 / 1.2838017$

Publication date:

2008

Document Version

Publisher's PDF, also known as Version of record

Link back to DTU Orbit

Citation (APA):

Michelena, M. D., Oelschlägel, W., Arruego, I., del Real, R. P., Mateos, J. A., \& Merayo, J. M. G. (2008).

Magnetic giant magnetoresistance commercial off the shelf for space applications. Journal of Applied Physics, 103(7), 07E912. https://doi.org/10.1063/1.2838017

\section{General rights}

Copyright and moral rights for the publications made accessible in the public portal are retained by the authors and/or other copyright owners and it is a condition of accessing publications that users recognise and abide by the legal requirements associated with these rights.

- Users may download and print one copy of any publication from the public portal for the purpose of private study or research.

- You may not further distribute the material or use it for any profit-making activity or commercial gain

- You may freely distribute the URL identifying the publication in the public portal 


\title{
Magnetic giant magnetoresistance commercial off the shelf for space applications
}

\author{
M. D. Michelena, ${ }^{1, a)}$ W. Oelschlägel,${ }^{2}$ I. Arruego, ${ }^{1}$ R. P. del Real, ${ }^{1}$ J. A. D. Mateos,${ }^{1}$ and \\ J. M. Merayo ${ }^{2}$ \\ ${ }^{1}$ Space Sciences and Space Programmes, INTA-Instituto Nacional de Técnica Aeroespacial, Torrejón de \\ Ardoz, Spain \\ ${ }_{2}^{2}$ Measurement and Instrumentation, DNSC-Danish National Space Center, DTU-Danmarks Tekniske \\ Universitet, Lyngby, Denmark
}

(Presented on 7 November 2007; received 15 September 2007; accepted 20 November 2007; published online 13 February 2008)

\begin{abstract}
The increase of complexity and miniaturizing level of Aerospace platforms make use of commercial off the shelf (COTS) components constitute a plausible alternative to the use of military or rad-tolerant components. In this work, giant magnetoresistance commercial sensors are studied to be used as COTS, the next missions to be launched in the framework of the Spanish National Space Program: OPTOS and SEOSAT. This technology of magnetic sensors is interesting due to their high operating range up to $2 \mathrm{mT}$ and the high temperature dynamic range from -50 up to $150{ }^{\circ} \mathrm{C}$. However, in contrast, it presents high hysteresis and nonlinearity, temperature dependence, and poor repeatability. To improve the hysteretic, nonlinear and nonrepetitive behavior, a method consisting of a combination of reset and biasing has been designed and implemented for the $\pm 75 \mu \mathrm{T}$ linear region centered around 300-375 $\mu \mathrm{T}$ biasing field. (C) 2008 American Institute of Physics. [DOI: $10.1063 / 1.2838017]$
\end{abstract}

\section{INTRODUCTION}

The conservative Aerospace sector traditionally used old and well experimented components in its developments. The utilization of brand new technologies in commercial off the shelf (COTS) for space missions, which normally have the added values of low mass, volume, and power consumption, started in the 1990s and was associated with small amateur satellites. However, nowadays, most of the Space Agencies are involved in the validation of COTS (Ref. 1) for their missions based mainly in three reasons:

1 COTS are more economic than highly qualified components. The price of a certain component increases linearly with the qualification level guaranteed by the manufacturer. Roughly speaking it can be said that military components are one order of magnitude more expensive than commercial components and rad-hardened components are one order of magnitude more expensive than military components.

2 The offer of COTS is wider and the delivery time of COTS is much lower than that of the highly qualified components.

3 The continuity in the production lines makes them very reliable and part of a continuous and fast improvement.

In the present work, we study the feasibility of using giant magnetoresistance (GMR) COTS for space applications. The GMR effect was discovered in 1988 and the first commercial devices appeared in the market in 1995 by NVE. ${ }^{2}$ Nowadays, the use of GMR switches is relatively common (detection of the speed and position of electric mo-

\footnotetext{
${ }^{a)}$ Electronic mail: diazma@inta.es.
}

tors shafts or bearings, automotive transmission gear speeds, axle shaft speed in antilock braking systems, and linear gear tooth position), but no previous work was done in the validation of these COTS for space applications.

GMR technology is a good alternative for measurements of the magnetic field in the range of millitesla because they have a higher magnetic field dynamic range (several millitesla) than anisotropic magnetoresistance (AMR) sensors, for instance, and a high temperature range too $(-50$, $\left.150{ }^{\circ} \mathrm{C}\right)$. However, in contrast, they present higher hysteresis, nonlinearity, and they have no sense determination. Previous works focused on the improvement of the response of these sensors ${ }^{3,4}$ using an ac biasing method both with a sine wave $^{3}$ and a square wave ${ }^{4,5}$ exciting signal to improve the linearity and the hysteresis behavior of the sensors response obtaining a sensitivity sensor with a linearity error of about $3 \%$ in the range of about $\pm 375 \mu \mathrm{T}$, a hysteresis of $0.5 \%$ of full scale, and a power spectral density (PSD) of about $35 \mathrm{nT}$ $\mathrm{rms} / \sqrt{\mathrm{Hz}}$ at $1 \mathrm{~Hz}$.

In this study, we analyzed the response curve of GMR COTS with different bias fields and the variation of the derivative of the response curve. This has been done to choose a working point to have the best linearity in the region of zero magnetic field.

\section{RESULTS AND DISCUSSION}

GMR commercial sensors first taken into consideration are manufactured by Hitachi and NVE sensors. We focused on NVE sensors as the Hitachi sensors are explicitly not recommended for radiation proof. ${ }^{6}$ The devices under study are the AA002, AAH002, and AAL002 by NVE chosen because of their higher sensitivities compared to other GMR 


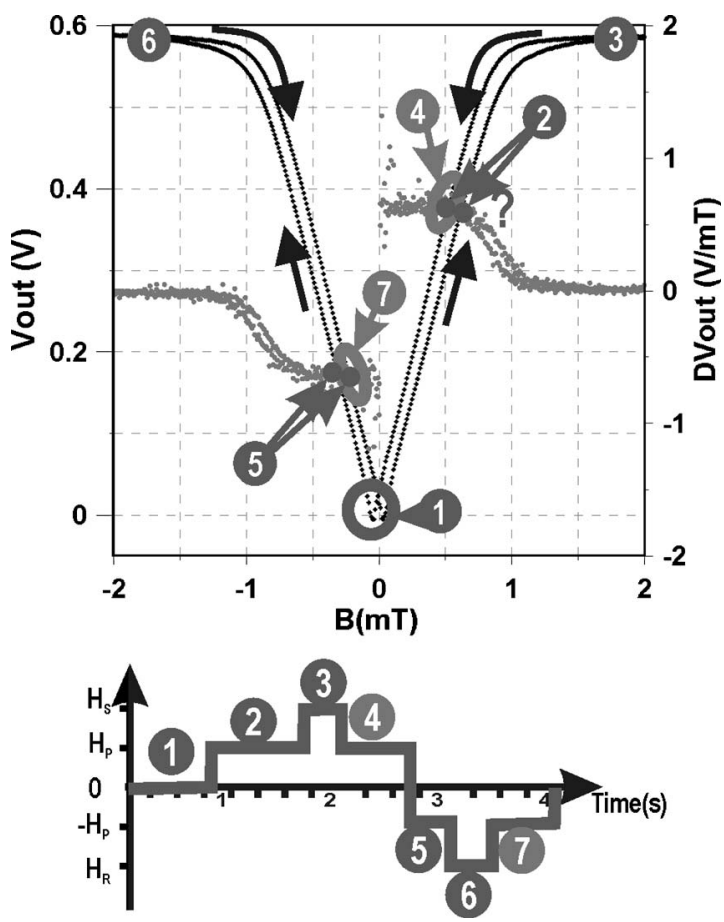

FIG. 1. Response curve of AA002 GMR sensor (black) and its magnetic field derivative (gray). The time diagram represents the set/reset routine.

sensors of the same families. This kind of technology has been radiated to disregard possible harmful effects in the components. Moreover, no effect was observed in their parameters.

GMR is an even effect, which implies that for the more important space applications position and angular sensors and Earth field sensing a biasing mechanism is necessary. In particular, the experiments designed for OPTOS and SEOSAT consist in field sensing devices with different complexity and magnetic field requirements. In both cases, they are not designed to map the Earth's magnetic field but, for instance, to detect quick changes $(\sim 3 \mu \mathrm{T})$ in a magnetic field of the order of $10 \mu \mathrm{T}$. In this work, a bias field $B_{B}$ is used for the sense determination and for linearization of the response. To get the most appropriate value of the bias field (the working point), the external field derivative of the response curve is studied. The bias field chosen is the central value of the constant region of the derivative (Fig. 1). This region corresponds to the field range of linear behavior but it still has some hysteresis. To solve this problem, a set/reset mechanism has been implemented.

Figure 1 shows the raw response curve of the AA002 sensor for a magnetic field range of $\pm 2 \mathrm{mT}$. It can be seen how the curve splits into two branches for positive and negative values of the applied field. As a direct consequence of this curve, the magnetization of the GMR multilayer and, thus, its output highly depends on the former state. That uncertainty can be avoided if the sensor is restarted for each measurement. In this principle lies the set/reset mechanism designed for the GMR sensors. In the presence of an external magnetic field, the sensor shows a nonunivocally defined output in a range within the two branches of the response curve of Fig. 1. If, in contrast, the sensor is previously mag-

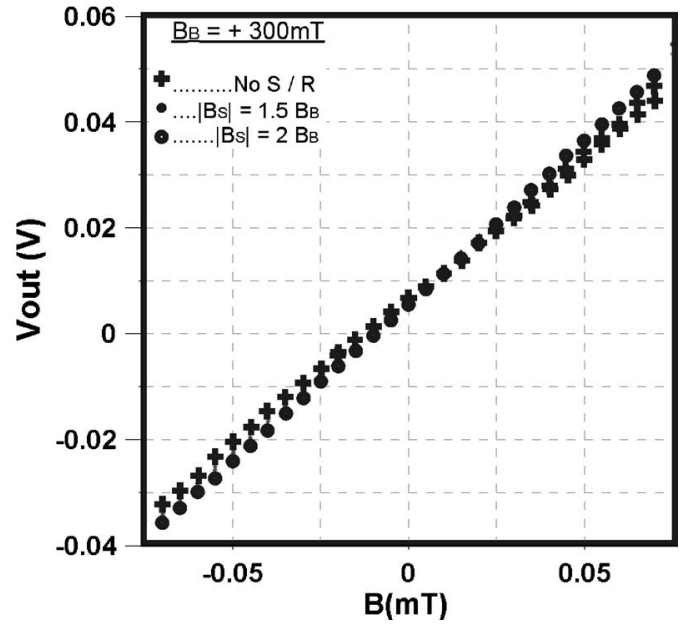

FIG. 2. Response curve of AAL002 by NVE in the $75 \mathrm{mT}$ range.

netized to state " 3 " (saturated), the effect of the external field is unique. The magnetization follows the demagnetizing curve, which besides, coincides with the highest sensitivity. If this is done in a routine and applying a bias field in both senses, the sensor improves its linearity and the asymmetric behavior of the sensor is avoided. The dc routine is shown in Fig. 1. However, at low frequencies, the signal to noise ratio is very poor because the sensor is affected by white noise and $1 / f$ noise. When the routine is developed at $1 \mathrm{kHz}$, the $1 / f$ contributions decreases and PSD is below $10^{-7} \mathrm{~V} / \sqrt{\mathrm{Hz}}$.

Measurements with this routine are made for the three sensors under study. To do so, an external wire is used so as not to change the temperature of the sensing multilayer. This is very important because of the dependence of the behavior of the response with the temperature. As a consequence, AA002 sensor is disregarded as it presents higher hysteretic behavior than AAL002 and AAH002 is disregarded because it has a lower dynamic range and the linearity in this range is poorer than other AMR COTS sensors.

The set field is also varied until the saturating field (Fig. 2 ). The data with crosses curve correspond to the measurement without any set/reset mechanism. Its coefficient of determination $R^{2}$ is very poor: 0.99819 . The dotted curve corresponding to a set field of 1.5 times that of biasing, has an improvement of $R^{2}: 0.99917$ and the last one, the circles curve, corresponding to saturating set values has a $R^{2}$ of 0.999 957. The sensitivity also increases slightly with the increase of the value of the set field. Regarding the hysteresis, the curve without set/reset mechanism has a maximum deviation by hysteresis of $3 \%$ while the other two curves is below $2 \%$.

An implementation of this technology for the determination of future applications for space is being developed in the frame of OPTOS project, which is a $10 \times 10 \times 30 \mathrm{~cm}^{3}$ Picosat devoted to be technological test bed. ${ }^{7}$ The block diagram of the proximity electronic for OPTOS mission can be seen in Fig. 3. The electronic circuit designed with components that have a Rad Hard version, consists of two main parts: The first part is devoted to amplify and to condition the GMR outputs, to measure the temperature for thermal calibration, and to multiplex the outputs. The second consists in 


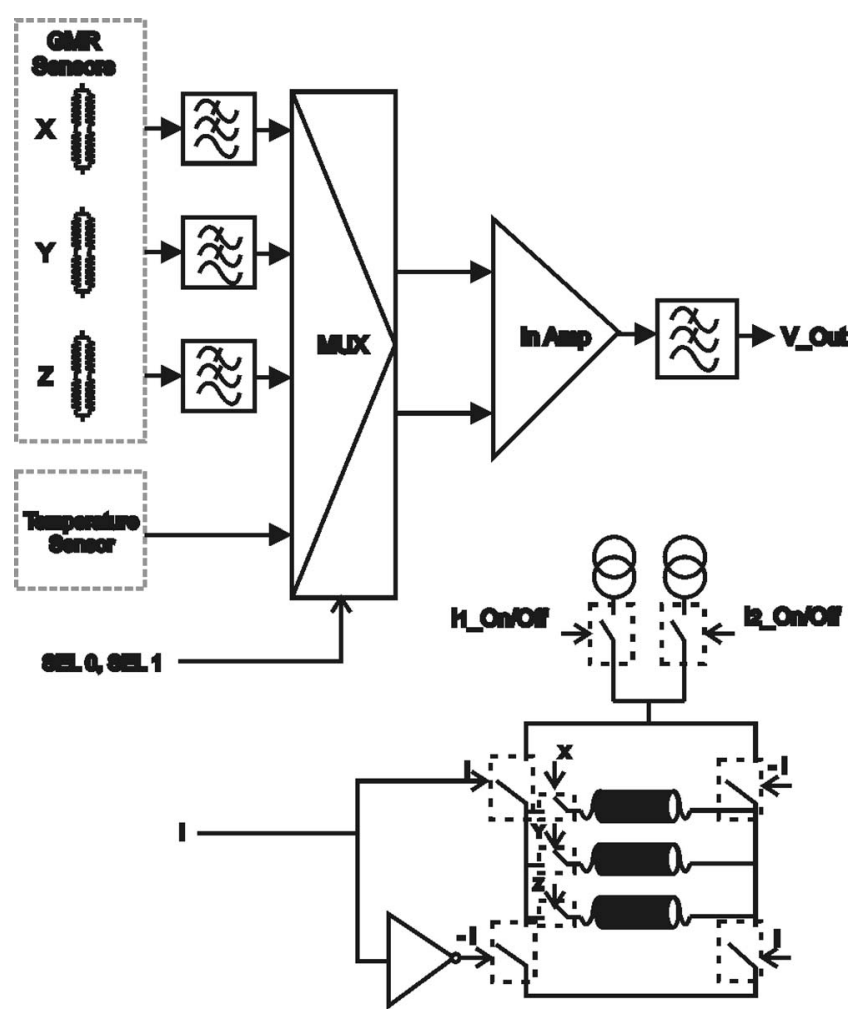

FIG. 3. Block diagram of the proximity electronic.

the set/reset and biasing circuitry for the three different axes. It is based on three coils (one per axis) that are driven with precision current source circuits, stabilized in temperature. There are two of these sources, so as to obtain the necessary current values for the generation of the bias field $B_{B}$ and set/reset field $B_{S}$. Finally, an $H$-bridge configuration is used to control the sense of the current through the coils, allowing $\pm B_{B / S}$ to be obtained. The duration of set and reset fields is $0.2 \mathrm{~ms}$ while the biasing fields are applied during $0.4 \mathrm{~ms}$. The data are taken when the bias fields are stable and the resulting value of the magnetic field is the semidifference of the two values corresponding to the bias fields in opposite senses of the measuring direction.

\section{CONCLUSIONS}

In the present work, the viability of using GMR COTS for space applications has been studied. To improve the behavior of the commercially available sensors, a novel method of combined set/reset and magnetic bias has been designed and tested. As a result, the linearity and the sensitivity of the sensor are increased and the hysteretic behavior is minimized up to $2 \%$ in the range of $\pm 75 \mu \mathrm{T}$ (in-orbit Earth magnetic field). In parallel, in the frame of OPTOS project, the COTS are being irradiated up to the radiation level of the mission, up-screened, and it has been designed and manufactured a robust (Rad Hardened) proximity electronic.

\section{ACKNOWLEDGMENTS}

This work has been partially financed by MEC Projects OPTOMAG-MANTIS ESP2005-05278 and MAGNETITA ESP2006-28460-E.

${ }^{1}$ K. L. Makovec, A. J. Turner, and C. D. Hall, AAS/AIAA Astrodynamics Specialists Conference, Quebec City, Canada, August 2001 (unpublished), Paper No. AAS 01-311.

${ }^{2}$ NVE Corporation GMR Sensor Catalog, pp. 14-19.

${ }^{3}$ P. Ripka, M. Tondra, J. Stokes, and R. Beech, Sens. Actuators, A 76, 227 (1999).

${ }^{4}$ M. Vopálenský, P. Ripka, and A. Platil, Sens. Actuators, A 106, 38 (2003). ${ }^{5}$ M. Vopálenský, P. Ripka, J. Kubík, and M. Tondra, Sens. Actuators, A 110, 254 (2004)

${ }^{6}$ Magnetic Compass Sensor HM55B Datasheet 19-SM-001-2, Chap. 9.2, p. 9.

${ }^{7}$ See: www.inta.es 